\title{
Airway T cells protect against RSV infection in the absence of antibody
}

\author{
E Kinnear ${ }^{1}$, L Lambert $^{1}$, JU McDonald ${ }^{1}$, HM Cheeseman ${ }^{1}$, LJ Caproni ${ }^{2}$ and JS Tregoning ${ }^{1}$
}

Tissue resident memory $\mathrm{T}$ (Trm) cells act as sentinels and early responders to infection. Respiratory syncytial virus (RSV)-specific Trm cells have been detected in the lungs after human RSV infection, but whether they have a protective role is unknown. To dissect the protective function of Trm cells, BALB/c mice were infected with RSV; infected mice developed antigen-specific $\mathrm{CD} 8^{+}$Trm cells $\left(\mathrm{CD} 103^{+} / \mathrm{CD}^{\circ} 9^{+}\right)$in the lungs and airways. Intranasally transferring cells from the airways of previously infected animals to naïve animals reduced weight loss on infection in the recipient mice. Transfer of airway CD8 cells led to reduced disease and viral load and increased interferon- $\gamma$ in the airways of recipient mice, while CD4 transfer reduced tumor necrosis factor- $\alpha$ in the airways. Because DNA vaccines induce a systemic T-cell response, we compared vaccination with infection for the effect of memory CD8 cells generated in different compartments. Intramuscular DNA immunization induced RSV-specific CD8 T cells, but they were immunopathogenic and not protective. Notably, there was a marked difference in the induction of Trm cells; infection but not immunization induced antigen-specific Trm cells in a range of tissues. These findings demonstrate a protective role for airway CD8 against RSV and support the need for vaccines to induce antigen-specific airway cells.

\section{INTRODUCTION}

Respiratory syncytial virus (RSV) is the leading cause of morbidity from lower respiratory tract viral infection in infants and children age $<5$ years. ${ }^{1}$ There is as yet no RSV vaccine, and while a number of candidates are in late stages of clinical trials, the ideal immune correlates of protection are not fully characterized. The main target of vaccine-induced adaptive immunity has traditionally been antibody. Antibody is a key correlate of protection against RSV, for example, maternal antibody levels closely correlate with the risk of RSV infection in the infant. ${ }^{2}$ But protection can occur in the absence of detectable serum antibodies, ${ }^{3}$ and infection can occur when serum-neutralizing antibodies are high. ${ }^{4}$ This suggests a role for protective T-cell immunity, supported by the observation that children with defective $\mathrm{T}$-cell responses were unable to clear the virus for several months and suffered from more severe disease. $^{5}$

Their protective capacity should make the induction of CD8 $\mathrm{T}$ cells an attractive goal for an RSV vaccine; and yet, there is a substantial body of evidence suggesting that excess T-cell responses can be detrimental. ${ }^{6-8}$ One possibility is that the wrong types of $\mathrm{T}$ cells, either functionally or spatially, are being induced. Memory T cells can be phenotypically characterized by their cell surface marker expression, with different subsets behaving differently on re-exposure to the pathogen. ${ }^{9}$ Recently, a novel population of tissue resident memory $\mathrm{T}$ ( $\mathrm{Trm}$ ) cells has been defined. These cells move from the circulation to the tissues, leading to the upregulation of $\mathrm{CD} 69$, the downregulation of S1P1, and normally the upregulation of the integrin $\mathrm{CD} 103$, which leads to their retention in the tissue. ${ }^{10}$ Functionally, these cells are primed to respond more rapidly to pathogen, with 20 times higher affinity for antigen than effector memory $\mathrm{T}$ cells, allowing them to detect cells expressing low levels of antigen, such as those in the early stages of infection. ${ }^{11}$ Once a pathogen is detected, Trm cells contribute to the early immune response by secreting cytokines such as interferon gamma $(\text { IFN } \gamma)^{12}$; for example, influenza-specific lung-resident $\mathrm{T}$ cells respond rapidly upon reactivation, producing multiple cytokines. ${ }^{13}$ It has recently been shown that the presence of RSV-specific CD8 ${ }^{+} \mathrm{T}$ cells in the lungs, but not the blood, of human adults correlates with less severe disease upon RSV challenge infection. ${ }^{14}$ Less research has been performed on

${ }^{1}$ Mucosal Infection and Immunity Group, Section of Virology, Department of Medicine, Imperial College London, St Mary's Campus, London, UK and ${ }^{2}$ Touchlight Genetics, Morelands \& Riverdale Buildings, Hampton, UK. Correspondence: JS Tregoning (john.tregoning@imperial.ac.uk)

Received 12 August 2016; accepted 2 April 2017; published online 24 May 2017. doi:10.1038/mi.2017.46 
$\mathrm{CD} 4{ }^{+}$Trm in the lungs, but they have been shown to have a protective role in influenza ${ }^{15}$ and Nippostrongylus brasiliensis. ${ }^{16}$

The aim of this study was to investigate the functional role of $\mathrm{Trm}$ in protection against RSV infection. We demonstrate for the first time that transferring airway CD4 or CD8 cells is sufficient to protect against disease after RSV infection. We then compared the effect of memory CD8 cells generated after lung infection or systemic vaccination and saw that systemic DNA vaccines induce pathogenic but not protective CD8 T cells. Comparing vaccination and infection, we see significant differences in the localization and type of antigen-specific CD8 cells, which may contribute to their different effects. From this, we conclude that airway-resident $\mathrm{T}$ cells are sufficient to protect against RSV and their induction should be a goal of vaccination.

\section{RESULTS}

Prior RSV infection protects against subsequent infection and induces both RSV-specific antibody and CD8 T cells Intranasal (i.n.) infection with wild-type RSV protects against subsequent exposures ${ }^{17}$ and antibody has a role in this protection, but the role of $\mathrm{T}$ cells is unclear. The aim of the study was to investigate the role of lung-resident $\mathrm{T}$ cells in protection against RSV infection. The response to RSV was compared after one, two, or three exposures to RSV, each 21 days apart. As expected, mice infected for the first time with RSV lost significantly more weight (Figure 1a), had more viral load on day 4 after infection (Figure 1b), and significantly lower anti-RSV immunoglobulin $\mathrm{G}(\mathrm{IgG})$ in serum after infection (Figure 1c). To distinguish between circulating and local cells, mice were pretreated with intravenous antibody prior collecting organs. CD8 T cells were recruited into both the lungs (Figure 1d) and airways (Figure 1e), peaking at day 8 of mice infected with RSV for the first time. There were significantly more total CD8 cells in the lungs or bronchoalveolar lavage after a single infection than after two or three infections. However, the proportion of CD8 cells that were Trm cells (defined as $\mathrm{CD}^{+} 9^{+}$and $\mathrm{CD}_{103}{ }^{+}$) was significantly greater in the lungs (Figure 1f) and airways (Figure 1g) of mice previously exposed to RSV than primary infection at days 4 and 8 after infection. This combination of markers has been used to identify Trm after viral infection in both human ${ }^{14}$ and murine studies, ${ }^{18}$ though it may also pick up some effector cells at the acute time points. Interestingly, the proportion of $\mathrm{CD} 69^{+} / \mathrm{CD} 103^{+}$cells was the same 21 days after the first, second or third exposure to RSV-rising to approximately $10 \%$ of CD8 cells after one exposure or contracting to the same proportion after two or three exposures. Of the $\mathrm{CD} 69^{+} / \mathrm{CD} 103^{+} \mathrm{CD} 8 \mathrm{Trm}$ cells, a high proportion were specific for the immunodominant peptide of the RSV M protein (Figure 1h,i). The proportion that was pentamer positive were significantly greater after re-exposure to RSV. There were no differences seen in the response after the second or third exposure to RSV.
Therefore, RSV-specific Trm are induced in the lungs and airways after infection.

\section{Airway cell transfer reduces disease after RSV infection}

The aim of the study was to define the role of airway cells in protection against RSV disease. Mice were infected with RSV, and 4 weeks later, $\mathrm{T}$ cells were depleted with antibody prior to reinfection with RSV. Depleting $\mathrm{CD}^{+}{ }^{+}$or $\mathrm{CD} 4^{+}$led to a significant increase in early weight loss compared with the RSV-RSV group, indicating that they have a role in the reduction of disease (Figure 2a). However, these mice had antiRSV antibody that may mask any protective effect of T cells. We used transfer studies to test cells in isolation. Donor mice were infected two times with RSV or sham infected with phosphatebuffered saline (PBS); 3 weeks after the second infection, mice were culled and cells were collected from the airways or spleens. In all, $10^{6}$ cells were instilled into the airways of naive animals, of these $22 \%$ were lymphocytes, and of the CD8 T cells transferred, 25\% were RSV-specific Trm accounting for $10^{4}$ cells transferred (see Supplementary Figure S1 online). One day after cell transfer, recipient animals were infected with RSV. Mice receiving cells from the airways of RSV exposed animals lost significantly less weight on days 6 and 7 compared with mice receiving splenocytes from RSV-exposed animals or cells from either the airways or spleens of PBS-treated animals (Figure 2b). There were significantly more CD8 (Figure 2c) and CD4 (Figure 2d) Trm in mice receiving cells from the airways of RSV-infected animals, 7 days after infection of the recipient mice. To determine the fate of transferred airway cells from naïve or RSV infected, they were labeled with cell trace violet prior to i.n. transfer into naïve animals. Recipient mice were infected with RSV 1 day after cell transfer and then culled 2 days after RSV infection. Labeled cells were detectable at a low frequency in the airways of all mice, with significantly more cells in the airways of RSV-infected animals receiving cells from RSV donors (see Supplementary Figure S1D).

To determine the relative contribution of CD4 and CD8 cells in protection against disease following RSV infection, airway cells from previously RSV-infected mice were sorted prior to i.n. transfer to naïve mice (see Supplementary Figure S2). Both CD4 and CD8 cell transfer reduced weight loss compared with control mice (Figure 2e). There were, however, phenotypic differences between mice receiving CD4 or CD8 cells. Mice that received CD8 cells had significantly lower viral load on day 4 of infection with RSV $(P<0.01$, Figure 2f $)$ and significantly greater IFN $\gamma$ in the airways on day 1 of infection $(P<0.01$, Figure 2g). Strikingly, transfer of $\mathrm{CD} 4{ }^{+}$cells led to a significant reduction in airway tumor necrosis factor alpha (TNF $\alpha)$ $(P<0.05$, Figure $2 \mathbf{h})$, but no effect on IFN $\gamma$ or viral load. Transferring sorted cells from the airways of PBS-exposed animals had no protective effect (Figure 2i). Curiously, transfer of unsorted cells from the airways of RSV-infected mice also reduced weight loss but without an impact on viral load, IFN $\gamma$, or TNF. From this, we conclude that airway CD8 are protective against reinfection and airway $\mathrm{CD} 4$ can reduce disease. 
a

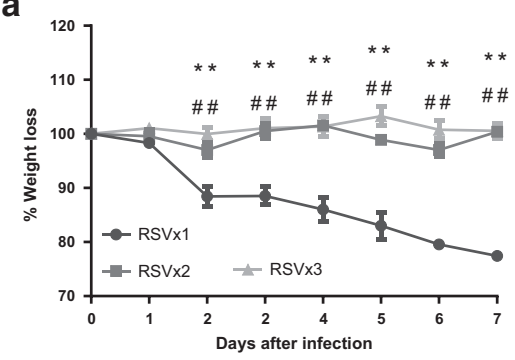

d

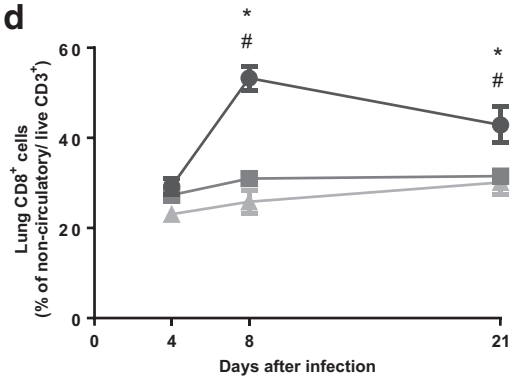

e

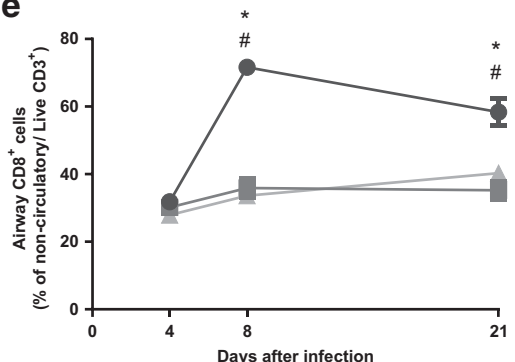

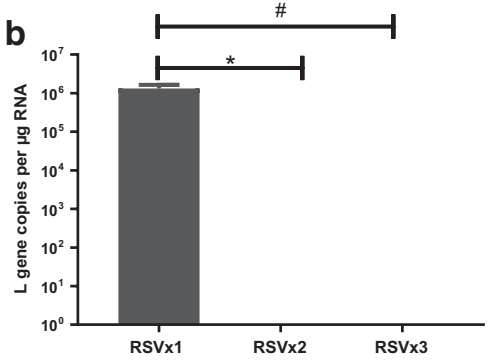

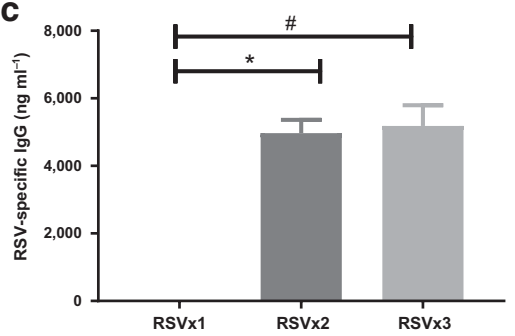

f

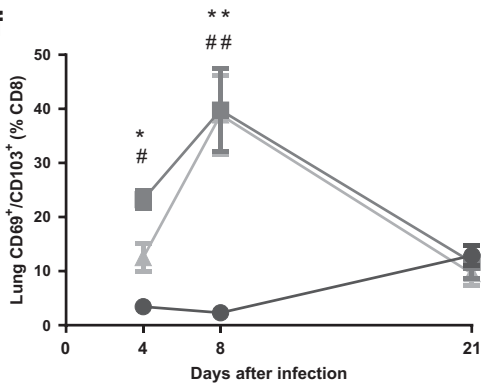

h

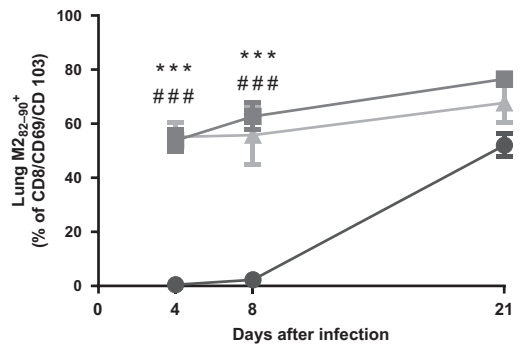

i

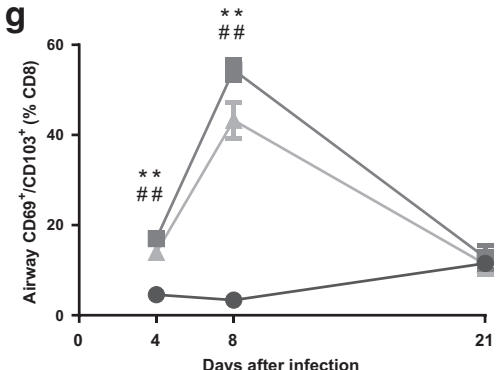

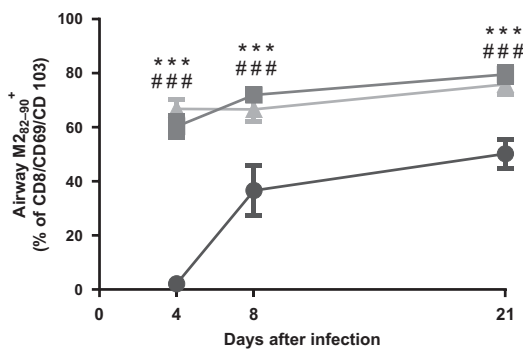

Figure 1 Prior respiratory syncytial virus (RSV) infection protects against subsequent infection and induces both RSV-specific antibody and CD8 T cells. Mice were infected with $10^{5}$ plaque-forming units RSV intranasally in $100 \mu \mathrm{l}$ once (blue circles), twice (red squares), or three (green triangles) times with RSV, with a 21-day gap between exposures. (a) Weight was measured daily after infection. (b) Viral load was measured on day 4 after infection. (c) Anti-RSV antibody responses were measured on day 7 after infection. (d, e) $\mathrm{CD} 8^{+} \mathrm{T}$ cells that were $(\mathbf{f}, \mathbf{g})$ resident memory $\mathrm{T}$ cells: $\mathrm{CD} 69^{+} / \mathrm{CD} 103^{+}$and $(\mathbf{h}, \mathbf{i})$ antigen specific in the $(\mathbf{d}, \mathbf{f}, \mathbf{h})$ lungs or $(\mathbf{e}, \mathbf{g}, \mathbf{i})$ airways, respectively, were measured by flow cytometry on days 4,8 , and 21 after infection. Points/

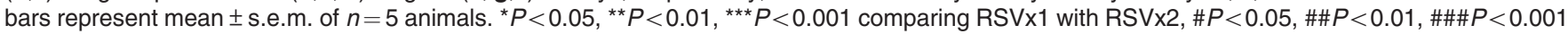
comparing RSVx1 with RSVx3. A full color version of this figure is available at the Mucosal Immunology journal online.

\section{Vaccines inducing systemic $\mathrm{T}$ cells do not protect against RSV infection}

Having observed that T-cell transfer reduced disease after RSV infection, we wished to test whether vaccination could induce T-cell-mediated protection. DNA vaccines have been shown to induce strong cellular responses in the systemic compartment ${ }^{19}$ and so we used them to test whether vaccine induced anti-RSV $\mathrm{T}$ cells could protect against RSV disease in mice. Mice were immunized intramuscularly with $5 \mu \mathrm{g}$ plasmid encoding RSV M2 in a prime boost-boost regime. Three weeks after the second immunization, mice were infected with RSV. RSV M2immunized mice lost more weight, more rapidly than untreated mice (Figure 3a). DNA immunization reduced the viral load at day 4 after infection but not to the same magnitude as previous infection with RSV (Figure 3b). Immunization with RSV M2 induced a significant population of RSV M2-specific CD8 cells; this was greater than in animals previously infected with RSV (Figure 3c). But DNA vaccination induced significantly fewer RSV-specific CD8 cells expressing Trm markers (Figure 3d). The DNA vaccine-induced RSV-specific cells were highly inflammatory, producing significantly more TNF $\alpha$ (Figure 3e) or IFN $\gamma$ (Figure 3f) than cells from naïve or previously exposed mice. It has been previously been demonstrated that excess CD8 cells in the airways can lead to enhanced disease in RSV. ${ }^{6,8}$ We wished to determine whether DNA vaccine-induced T cells were causing disease. When CD8 cells were depleted using antibody during RSV infection in spite of an increase of viral load (Figure 3g), there was a significant reduction in weight loss (Figure 3h). From this, we conclude that systemically induced $\mathrm{T}$ cells can cause disease rather than protect.

\section{Immunization and infection induce different antigen-specific $\mathrm{T}$ cell populations in different tissues} During infection, the proportion of airway CD8 T cells specific for the immunodominant M2 epitope was similar between RSV-infected and DNA-vaccinated animals, but there were significant differences in the proportion of those that expressed the Trm markers CD69 and CD103. To determine whether these differences occurred at the time of initial exposure to RSV antigens and whether there were differences in the cellular 
a

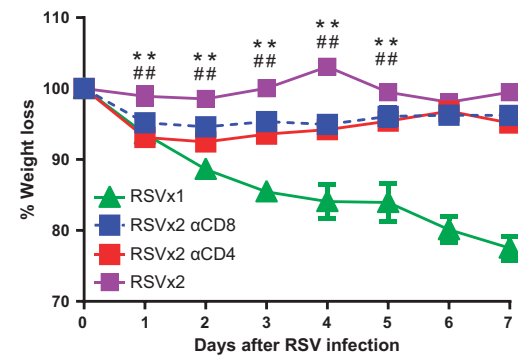

d

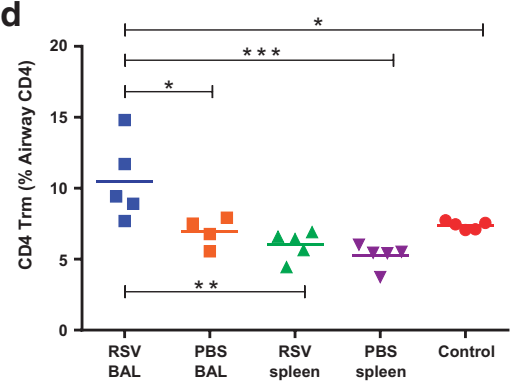

g

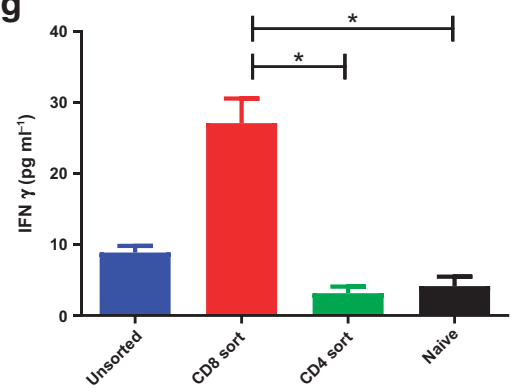

b

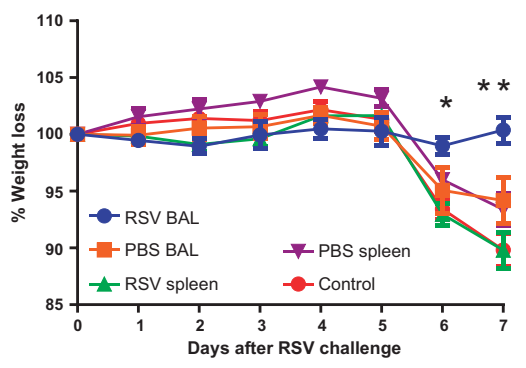

e

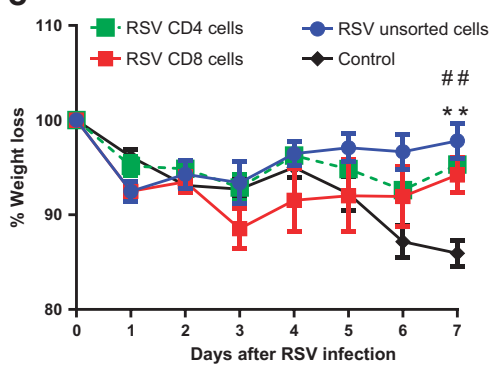

h

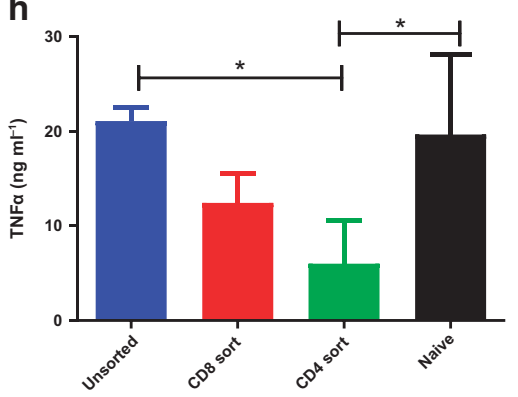

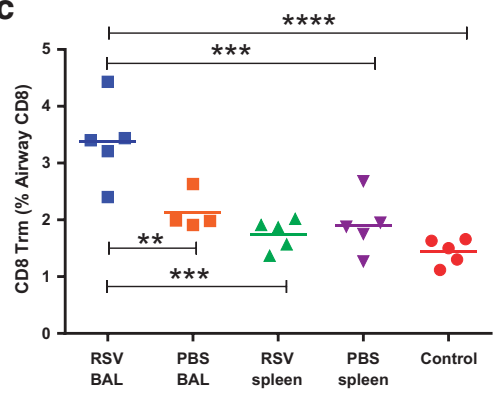

f

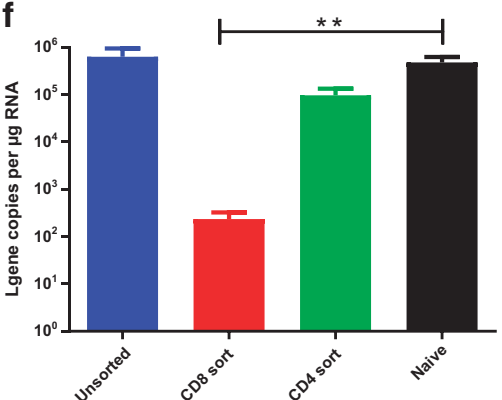

i

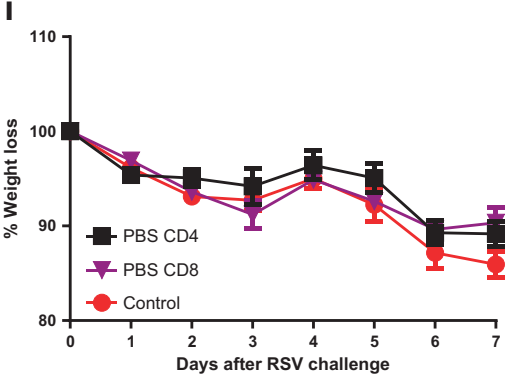

Figure 2 Airway cell transfer reduces disease after respiratory syncytial virus (RSV) infection. (a) BALB/c mice were infected with $10^{5}$ plaque-forming units (pfu) RSV; prior to reinfection, groups received either anti-CD8 or anti-CD4 antibodies on days $-1,+2$, and +5 after infection and the percentage of weight was measured. (b) Naïve mice received $10^{6}$ cells intranasally from the airways and spleens of RSV-exposed or phosphate-buffered saline (PBS)-exposed mice prior to infection with $10^{5}$ pfu RSV. (c) $\mathrm{CD}^{+}$and (d) $\mathrm{CD} 4^{+}$resident memory T (Trm) cells in the airways of recipient mice 7 days after RSV infection. Cells were sorted from airways of RSV-exposed mice prior to infection RSV. (e) Weight loss, (f) lung viral load at day 4 after RSV infection, (g) interferon gamma (IFN $\gamma$ ) and (h) tumor necrosis factor alpha (TNF $\alpha$ ) levels in the airways 1 day in recipient mice after infection. (i) Cells from PBS-exposed mice were sorted and transferred into mice before infection and weight loss. Points and bars represent $n=5$ animals \pm s.e.m. and ${ }^{\star} P<0.05,{ }^{* *} P<0.01,{ }^{* * *} P<0.001,{ }^{* * * *} P<0.0001$ as measured via one-way analysis of variance (ANOVA) with Tukey's multiple comparison post test. Weight loss significance was calculated by two-way ANOVA: for a significance is shown between RSVx2 and anti-CD4 (*) or anti-CD8 (\#); for $\mathbf{b}$ significance is shown between RSV brocheoalveolar lavage (BAL) and control (*); for e significance is shown between control and CD4 (\#) or CD8 ( $\left.{ }^{*}\right)$.

distribution, we compared PBS-immunized, RSV-infected and M2 DNA-immunized animals. Mice receiving DNA were immunized three times at 2-week intervals in the left anterior tibialis muscle in the hind limb. There were some differences in timing between exposure and recovery of cells: PBS or RSV groups were culled 7 weeks after the initial exposure, and DNAvaccinated mice were culled 3 weeks after the final exposure; all animals were culled and the samples were analyzed at the same time.

The phenotype of CD8 cells in the site of immunization (the left hind limb muscle and covering skin) was compared with the distal, right hind limb muscle and skin, airways, lung, blood, and spleen. No RSV-specific CD8 cells were detected after PBS delivery (Figure 4a). RSV infection led to populations of RSVspecific cells in all tissue compartments (right muscle was not measured owing to technical complications). DNA vaccination with a plasmid encoding the M2 gene induced a population of M2-specific CD8 cells in the immunized muscle (left) but not the distal, right muscle. DNA immunization induced a larger population of RSV-specific cells in the blood and lungs than the airways or spleen. RSV-specific cells in the lungs after vaccination may represent blood contamination because the lungs were not flushed prior to analysis. RSV infection induced a population of RSV-specific CD8 T cells in all tissues assessed. Strikingly, infection induced significantly more RSV-specific CD8 Trm cells in the lungs and skin sections than DNA vaccination (Figure $\mathbf{4 b}$ ). Therefore, infection and immunization induce qualitatively different cell populations recognizing the same epitope, with only infection leading to the induction of a protective CD8 T-cell subset in the lungs. 
a

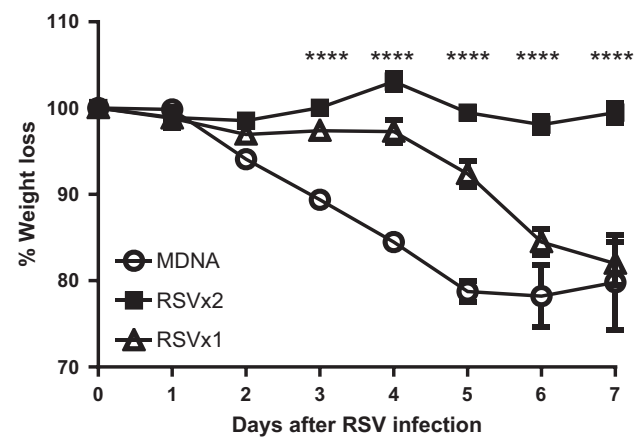

C

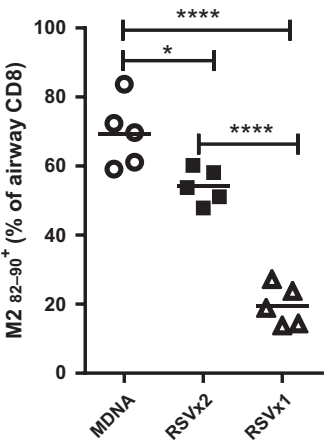

d

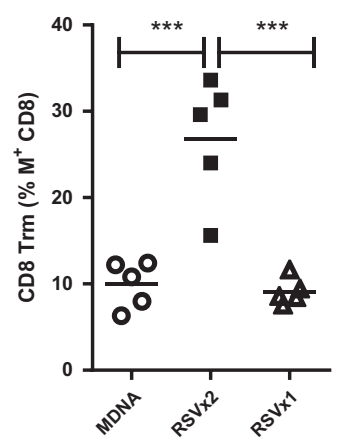

g

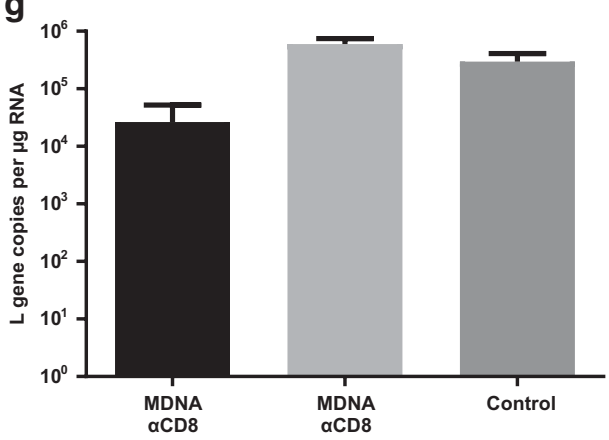

b
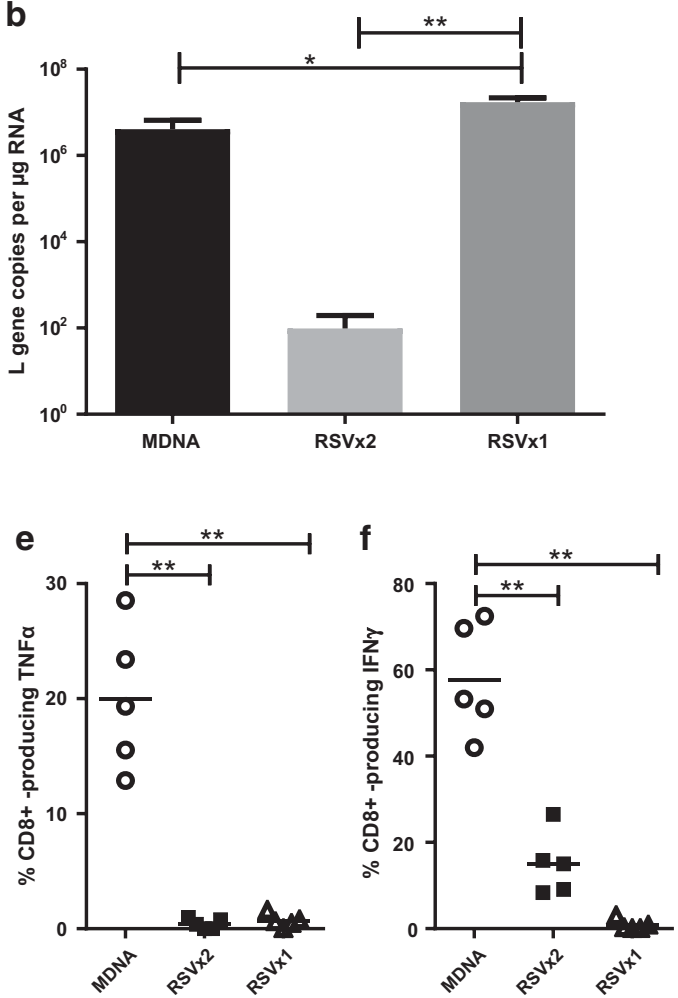

h

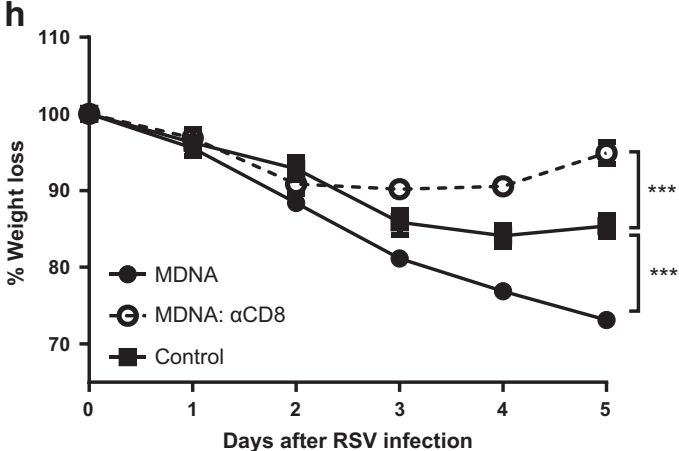

Figure 3 Vaccines inducing systemic T cells do not protect against respiratory syncytial virus (RSV) infection. BALB/c mice were intramuscularly vaccinated with electroporation using a three-dose regimen with $5 \mu \mathrm{g}$ RSV M DNA vaccine. Mice were infected with $10^{5}$ plaque-forming units RSV and compared with naïve (RSVx1) or previously exposed (RSVx2) animals; (a) weight loss after RSV challenge. (b) Lung RSV viral load at day 4 after

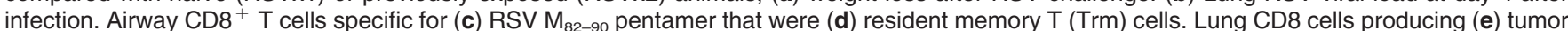
necrosis factor (TNF) or (f) interferon gamma (IFN $\gamma$ ). Mice were vaccinated three times with RSV M DNA prior to challenge CD8 cells were depleted. (g)

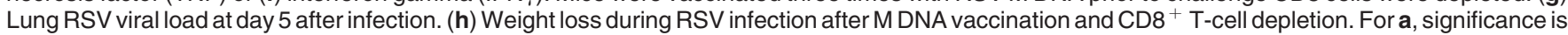
shown between RSVx2 and RSVx1 $\left(^{*}\right)$. Points and bars represent $n=5$ animals \pm s.e.m. and ${ }^{*} P<0.05,{ }^{* *} P<0.01,{ }^{* * *} P<0.001,{ }^{* * * *} P<0.0001$ as measured via one-way analysis of variance (ANOVA) with Tukey's multiple comparison post test. Weight loss significance was calculated by two-way ANOVA.

\section{DISCUSSION}

In the current study, we demonstrate for the first time that airway $\mathrm{T}$ cells are sufficient to protect against infection, even in the absence of antibody. Transferring CD8 T cells from mice previously infected with RSV to naïve animals reduced weight loss and viral load on exposure to RSV. This is an important finding because of the implication it has for RSV vaccine strategies. It ties in closely with a recent study which demonstrated that i.n. vaccination with a recombinant virus expressing RSV proteins was protective against infection and induced RSV-specific tissue Trm cells. ${ }^{20}$ However, caution needs to be taken with T-cell-targeting vaccines for RSV because the induction of CD8 $\mathrm{T}$ cells in the systemic compartment led to enhanced disease on exposure to RSV infection.

The transfer studies demonstrate that cells resident in the airways are protective against RSV infection. Functionally, there was an increase in IFN $\gamma$ in the airways after CD8 transfer, which may lead to the recruitment of other antiviral cells to the airway, though the CD8 cells themselves may be having a direct 

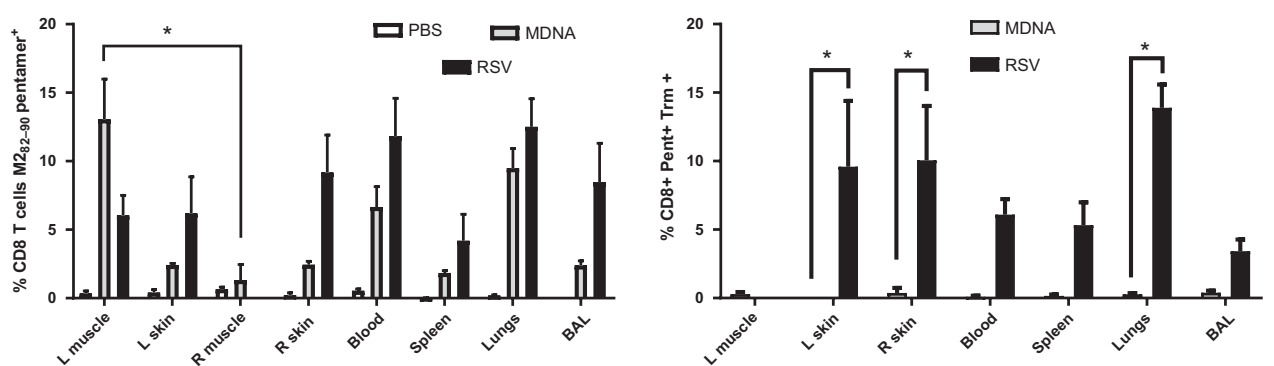

Figure 4 Immunization and infection induce different antigen-specific T cells populations in different tissues. BALB/c mice were sham inoculated with phosphate-buffered saline (PBS) intranasally (white bars) or infected with $10^{5}$ plaque-forming units respiratory syncytial virus (RSV) Strain A2 (black bars) or vaccinated with $5 \mu \mathrm{g}$ RSV M DNA (gray bars) in the left leg muscle in a prime boost-boost regimen with 2-week intervals. Seven weeks after the start of the study, all mice were killed. The left and right flank skin and muscle, blood, spleen, airway, and lung cells were analyzed. (a) CD8 ${ }^{+} \mathrm{T}$ cells specific for $\mathrm{M}_{82-90}$ pentamer ${ }^{+}$and (b) M-specific CD8 ${ }^{+}$T cells displaying resident memory $\mathrm{T}$ (Trm) cell markers. Points represent $n=5$ animals $\pm \mathrm{s}$.e.m. and ${ }^{*} P<0.05$ by multiple weight $t$-test.

antiviral effect. It was of note that transferring CD4 T cells from the airways reduced disease, without affecting viral load. There was a reduction in TNF $\alpha$ levels following CD4 T-cell transfer; we have previously shown that blocking TNF $\alpha$ reduces disease $^{21}$ and it may be that some of the CD4 cells transferred are regulatory $\mathrm{T}$ cells as we have shown that Treg depletion increases TNF $\alpha$ levels and disease. ${ }^{22}$ Total cell transfer reduced weight loss more than either cell alone, so either $\mathrm{T}$ cells are working in concert or there is a role for other cell types in the airways, for example, macrophages. Macrophages make up the majority of airway cells even after RSV infection and the transferred macrophages may also release cytokines locally and promote antiviral responses. ${ }^{23}$ Splitting the cells into different subsets prior to transfer would enable the identification of which memory or effector $\mathrm{T}$ cells are protective.

One of the striking findings was that, after infection, RSVspecific $\mathrm{T}$ cells were detectable in all tissues sampled. After infection, cells from the lungs or the skin had a greater propotion of $\mathrm{CD} 69^{+} / \mathrm{CD} 103^{+}$cells. The skin is a known site for tissue-resident cells, ${ }^{24}$ and it may be that effector cells induced in the lungs track to other tissues where they take on the characteristics of resident memory cells. It was of note that although RSV infection induces antigen-specific cells in the spleen, transferring splenocytes was not protective: a similar phenomenon was seen when protection was transferred with airway cells but not splenocytes from influenza-infected mice. ${ }^{15,25}$ It will be important to determine the factors that lead to the induction of Trm in the lungs and to determine whether these can be replicated artificially for vaccine strategies. In the skin, the cytokines transforming growth factor- $\beta$ and interleukin-15 are required for Trm development, ${ }^{26}$ but their role in lung Trm formation is not known.

One approach that has promise for the induction of resident memory cells is mucosal vaccination. It was striking that intramuscular DNA vaccination, while it did induce antigenspecific cells in the immunized tissue and the blood, did not induce tissue-resident memory cells in any site tested, even the site of immunization. Route has a significant effect on the qualitative response to DNA vaccination. ${ }^{27}$ Intranasal immunization with influenza DNA in a $100 \mu \mathrm{l}$ volume also led to the induction of airway-resident memory cells. ${ }^{28}$ Likewise, in a recent study, i.n. delivery of a recombinant viral vector expressing RSV $\mathrm{M}$ led to the induction of tissue-resident RSV-specific cells but only when delivered in a large enough volume to reach the lungs $(100 \mu \mathrm{l})$; intraperitoneal immunization with the same vaccine did not induce Trm. ${ }^{20}$ These studies suggest that the vaccine needs to get into the lungs to induce the correct type of memory cells and that responses in the upper airway are not the same as the lower airway. Vaccination at other mucosal sites can also lead to the induction of Trm, intravaginal immunization with human papillomavirus vectors expressing RSV antigen led to the induction of RSV specific Trm in the vagina, but when the same antigen was delivered intramuscularly, Trm were not induced. ${ }^{29}$ One potential strategy is to induce the formation of memory $\mathrm{CD}^{+}{ }^{+} \mathrm{T}$ cells in circulation that can then be 'pulled' to a local site ${ }^{30}$; however, we had limited success using this strategy to recruit antigen-specific $\mathrm{B}$ cells to the mucosa. ${ }^{31}$

Ultimately, an RSV vaccine needs to be effective in early life, when the bulk of severe disease happens. The infant immune response, in particular the cellular response, is different from that of adult immune response. We have previously shown that neonatal RSV infection primes for a immunopathogenic CD8 response, ${ }^{6,32}$ similar to the phenotype seen after DNA vaccination. How the immune environment in the neonatal lung differs from the adult lung with regards to the priming of T-cell responses is an important research topic for the development of optimum vaccines. Although RSV vaccines that are closest to the clinic are being developed for pregnant women with a view of passing on passive humoral immunity to the newborn, ${ }^{33}$ based on the data presented here, we would also advocate strategies that induce local $\mathrm{T}$ cells.

\section{METHODS}

Mouse immunization and infection. Six-to-8-week-old female BALB/ c mice were obtained from Harlan UK (Oxford, UK) and kept in specific-pathogen-free conditions in accordance with the United Kingdom's Home Office guidelines. All work was approved by the Animal Welfare and Ethical Review Board at Imperial College London. RSV A2 virus were grown using the human laryngeal carcinoma cell line, HEp-2. Viral titer was calculated by an immuno-plaque assay using biotinylated goat anti-RSV polyclonal antibody (AbD Serotec, 
Oxford, UK) to detect plaques. For infections, mice were anesthetized using isoflurane and infected i.n. with $10^{5}$ plaque-forming units RSV A2 in $100 \mu \mathrm{l}$. For DNA vaccination, mice were injected $5 \mu \mathrm{g}$ plasmid DNA expressing the M2-1 gene in $20 \mu \mathrm{l}$ intramuscularly into the left anterior tibialis muscle. Mice were DNA immunized three times, 2 weeks apart. In some studies, to distinguish between circulating and tissue-resident cells, mice were pretreated intravenously with $3 \mu \mathrm{g}$ antiCD45.2 (A700 fluorophore) in $200 \mu \mathrm{l} 3 \mathrm{~min}$ prior to killing. ${ }^{34}$

RSV viral load. Viral load in vivo was assessed by extracting RNA from frozen lung tissue disrupted in a TissueLyzer (Qiagen, Manchester, UK) using Trizol extraction and then converting it into cDNA. Quantitative reverse transcriptase-PCR was carried out using bulk viral RNA for the RSV L gene and mRNA using $900 \mathrm{~nm}$ forward primer ( $5^{\prime}$-GAACTCAGTGTAGGTAGAATGTTTGCA-3'), $300 \mathrm{~nm}$ reverse primer (5'-TTCAGCTATCATTTTCTCTGCCAAT- $\left.3^{\prime}\right)$ and $100 \mathrm{~nm}$ probe $\left(5^{\prime}\right.$-FAM-TTTGAACCTGTCTGAACAT-TAMRA- $\left.3^{\prime}\right)$ on a Stratagene Mx3005p (Agilent Technologies, Santa Clara, CA). L-specific RNA copy number was determined using a RSV L gene standard.

Semiquantitative antigen-specific enzyme-linked immunosorbent assay. Antibodies specific to RSV were measured in sera using a standardized enzyme-linked immunosorbent assay. ${ }^{35}$ MaxiSorp 96-well plates (Nunc, Thermo Scientific, Hemel Hempstead, UK) were coated with $1 \mu \mathrm{g} \mathrm{ml}^{-1} \mathrm{RSV}$ lysate or a combination of anti-murine lambda and kappa light-chain-specific antibodies (AbDSerotec, Oxford, UK) and incubated overnight at $4{ }^{\circ} \mathrm{C}$. Plates were blocked with $1 \%$ bovine serum albumin in PBS. Bound IgG was detected using horseradish peroxidase-conjugated goat anti-mouse IgG (AbD Serotec). A dilution series of recombinant murine IgG was used as a standard to quantify specific antibodies. Tetramethylbenzidine with $\mathrm{H}_{2} \mathrm{SO}_{4}$ as stop solution was used to detect the response, and optical densities were read at $450 \mathrm{~nm}$.

\section{Cell isolation}

Lungs, airway cells and spleen. Mice were culled using $100 \mu \mathrm{l}$ intraperitoneal pentobarbitone (20 mg dose, Pentoject, Animalcare, York, UK). Spleens, lung tissue and bronchoalveolar lavage were collected as previously described. ${ }^{36}$ Lungs and spleens were homogenized by passage through $100-\mu \mathrm{m}$ cell strainers, then centrifuged at $200 \mathrm{~g}$ for $5 \mathrm{~min}$. Supernatants were removed and the cell pellet was treated with red blood cell lysis buffer (ACK; $0.15 \mathrm{M}$ ammonium chloride, $1 \mathrm{~m}$ potassium hydrogen carbonate, and $0.01 \mathrm{~mm}$ EDTA, $\mathrm{pH}$ 7.2) before centrifugation at $200 \mathrm{~g}$ for $5 \mathrm{~min}$. The remaining cells were resuspended in RPMI 1640 medium with 10\% fetal calf serum, and viable cell numbers were determined by trypan blue exclusion.

Blood. For antibody-specified time points after immunization, blood samples were taken by tail vein bleed and sera were isolated after clotting by centrifugation. For cell isolation, blood was collected in heparinized capillary tubes (Hirschmann Laborgeräte, Eberstadt, Germany), followed by ACK lysis.

Skin and muscle recovery. Killed mice had both their legs shaved with electric clippers. A $2-\mathrm{cm}^{2}$ area of skin over each of the anterior tibialis muscles was excised and placed in a well containing Dulbecco's modified Eagle's medium $+10 \%$ fetal calf serum (D10) in a 12 -well plate kept on ice. The anterior tibialis muscles were also excised. Skin and muscle samples were chopped up into $3 \mathrm{~mm}^{3}$ sections. One milliliter of the digestion cocktail containing $12.5 \mu \mathrm{g} \mathrm{ml}^{-1}$ Liberase TL in SF media, DNAse at $200 \mu \mathrm{g} \mathrm{ml}^{-1}$, and hyaluronidase at $50 \mu \mathrm{g} \mathrm{ml}^{-1}$ was added to each sample as described previously. ${ }^{37}$ Samples were placed in a shaking block at $37^{\circ} \mathrm{C}$ for $1 \mathrm{~h}$ and then the digested sample was filtered through a $70-\mu \mathrm{m}$ cell strainer and centrifuged at $528 \mathrm{~g}$ for $5 \mathrm{~min}$. The supernatant was discarded and the cell pellet was resuspended in $5 \mathrm{ml} \mathrm{ACK} \mathrm{lysis} \mathrm{buffer} \mathrm{for} 5 \mathrm{~min}$. Also, $1.5 \times$ volume D10 was added and cells were again centrifuged at $528 \mathrm{~g}$ for $5 \mathrm{~min}$.

Airway cell transfer. Cells were collected from the bronchoalveolar lavage or spleen, washed, and resuspended in sterile PBS. Mice were anesthetized and $10^{6}$ cells in $100 \mu \mathrm{l}$ were delivered i.n. with a Gilson pipette (Dunstable, UK). Isolated cells from the airways were negatively sorted for $\mathrm{CD}^{+}{ }^{+}$and $\mathrm{CD} 8{ }^{+}$populations using the MACS $\mathrm{CD}^{+}$and $\mathrm{CD}^{+}{ }^{+}$T-cell isolation kits as per the manufacturer's instructions from $10^{7}$ airway cells using LS columns. The purity of isolated $\mathrm{CD}^{+}{ }^{+}$or $\mathrm{CD} 8{ }^{+}$cells were analyzed by flow cytometry. To track cells, prior to transfer $5 \mu \mathrm{M}$ Celltrace Violet dye (Invitrogen, Paisley, UK) was added to $10^{6}$ cells, they were incubated with gentle agitation, washed, and resuspended in sterile PBS.

Flow cytometry. Cells were stained with Fixable Violet Dead Cell Stain (Life Technologies, Paisley, UK), washed, suspended in Fc block (AntiCD16/32, BD) in PBS-1\% bovine serum albumin, and then stained with surface antibodies: RSV M2 82-90 Pentamer R-PE (Proimmune, Oxford, UK), CD3-FITC (BD, Oxford, UK), CD4-PE/Cy7 (BioLegend, San Diego, CA), CD8-APC-H7 (BD), CD69-APC (BioLegend), and CD103-PerCP Cy5.5 (BioLegend). Analysis was performed on an LSRFortessa flow cytometer (BD). Fluorescence minus one controls were used for surface stains.

Cytokine detection. Cytokine responses in the airway and lung cells after transfer and RSV infection were analyzed using a TH1/TH2 Group 1 Bio-Plex Pro Mouse Cytokine Assay Kit (Bio Rad, Watford, UK) according to the manufacturer's instructions.

Statistical analysis. Calculations as described in figure legends were performed using Prism 6 (GraphPad Software, La Jolla, CA).

SUPPLEMENTARY MATERIAL is linked to the online version of the paper at http://www.nature.com/mi

\section{ACKNOWLEDGMENTS}

E.K. was funded by an MRC CASE studentship MR/J006548/1 with financial support from Touchlight Genetics. We thank Dr A Walters (Oxford University) for purifying plasmid DNA. The study was funded in part by Touchlight Genetics, who were involved in all stages of the study conduct and analysis.

\section{AUTHOR CONTRIBUTIONS}

E.K., L.J.C., and J.S.T. designed the studies. E.K., L.L., J.U.M., and H.M.C. performed the experimental studies. E.K. and J.S.T. wrote the manuscript.

\section{DISCLOSURE}

Dr Caproni is an employee of Touchlight Genetics. The other authors declared no conflict of interest.

Official journal of the Society for Mucosal Immunology

\section{REFERENCES}

1. Tregoning, J.S. \& Schwarze, J. Respiratory viral infections in infants: causes, clinical symptoms, virology, and immunology. Clin. Microbiol. Rev. 23, 74-98 (2010).

2. Stensballe, L.G., Ravn, H., Kristensen, K., Meakins, T., Aaby, P. \& Simoes, E.A. Seasonal variation of maternally derived respiratory syncytial virus antibodies and association with infant hospitalizations for respiratory syncytial virus. J. Pediatr. 154, 296-298 (2009).

3. Piedra, P.A., Jewell, A.M., Cron, S.G., Atmar, R.L. \& Glezen, W.P. Correlates of immunity to respiratory syncytial virus (RSV) associatedhospitalization: establishment of minimum protective threshold levels of serum neutralizing antibodies. Vaccine 21, 3479-3482 (2003).

4. Habibi, M.S. et al. Impaired antibody-mediated protection and defective IgA B-cell memory in experimental infection of adults with respiratory syncytial virus. Am. J. Respir. Crit. Care Med. 191, 1040-1049 (2015). 
5. Hall, C.B. et al. Respiratory syncytial viral infection in children with compromised immune function. N. Engl. J. Med. 315, 77-81 (1986).

6. Tregoning, J.S., Yamaguchi, Y., Harker, J., Wang, B. \& Openshaw, P.J. The role of T cells in the enhancement of respiratory syncytial virus infection severity during adult reinfection of neonatally sensitized mice. J. Virol. 82, 4115-4124 (2008).

7. Graham, B.S., Bunton, L.A., Wright, P.F. \& Karzon, D.T. Role of T lymphocyte subsets in the pathogenesis of primary infection and rechallenge with respiratory syncytial virus in mice. J. Clin. Invest. 88, 1026-1033 (1991).

8. Openshaw, P.J. \& Tregoning, J.S. Immune responses and disease enhancement during respiratory syncytial virus infection. Clin. Microbiol. Rev. 18, 541-555 (2005).

9. Lauvau, G., Boutet, M., Williams, T.M., Chin, S.S. \& Chorro, L. Memory CD8 + Tcells: innate-like sensors and orchestrators of protection. Trends Immunol. 37, 375-385 (2016).

10. Mueller, S.N. \& Mackay, L.K. Tissue-resident memory T cells: local specialists in immune defence. Nat. Rev. Immunol. 16, 79-89 (2016).

11. Frost, E.L., Kersh, A.E., Evavold, B.D. \& Lukacher, A.E. Cutting edge: resident memory CD8 T cells express high-affinity TCRs. J. Immunol. 195, 3520-3524 (2015).

12. Schenkel, J.M., Fraser, K.A., Beura, L.K., Pauken, K.E., Vezys, V. \& Masopust, D. Tcell memory. Resident memory CD8 Tcells trigger protective innate and adaptive immune responses. Science 346, 98-101 (2014).

13. Purwar, R., Campbell, J., Murphy, G., Richards, W.G., Clark, R.A. \& Kupper, T.S. Resident memory T cells (T(RM)) are abundant in human lung: diversity, function, and antigen specificity. PLOS ONE 6, e16245 (2011).

14. Jozwik, A. et al. RSV-specific airway resident memory CD8 + T cells and differential disease severity after experimental human infection. Nat. Commun. 6, 10224 (2015).

15. Teijaro, J.R., Turner, D., Pham, Q., Wherry, E.J., Lefrancois, L. \& Farber, D.L. Cutting edge: tissue-retentive lung memory CD4 T cells mediate optimal protection to respiratory virus infection. J. Immunol. 187, 5510-5514 (2011).

16. Thawer, S.G. et al. Lung-resident $\mathrm{CD} 4(+) \mathrm{T}$ cells are sufficient for IL-4Ralpha-dependent recall immunity to Nippostrongylus brasiliensis infection. Mucosal Immunol. 7, 239-248 (2014).

17. Russell, R.F., McDonald, J.U., Ivanova, M., Zhong, Z., Bukreyev, A. \& Tregoning, J.S. Partial attenuation of respiratory syncytial virus with a deletion of a small hydrophobic gene is associated with elevated interleukin-1beta responses. J. Virol. 89, 8974-8981 (2015).

18. Wakim, L.M., Smith, J., Caminschi, I., Lahoud, M.H. \& Villadangos, J.A. Antibody-targeted vaccination to lung dendritic cells generates tissueresident memory CD8 $\mathrm{T}$ cells that are highly protective against influenza virus infection. Mucosal Immunol. 8, 1060-1071 (2015).

19. Tregoning, J.S. \& Kinnear, E. Using plasmids as DNA vaccines for infectious diseases. Microbiol. Spectr. 2, 1-16 (2014).

20. Morabito, K.M., Ruckwardt, T.R., Redwood, A.J., Moin, S.M., Price, D.A. \& Graham, B.S. Intranasal administration of RSV antigen-expressing MCMV elicits robust tissue-resident effector and effector memory CD8 + Tcells in the lung. Mucosal Immunol. 10, 545-554 (2016).

21. Tregoning, J.S. et al. The chemokine MIP1alpha/CCL3 determines pathology in primary RSV infection by regulating the balance of T cell populations in the murine lung. PLOS ONE 5, e9381 (2010).

22. Lee, D.C. et al. CD25 + natural regulatory Tcells are critical in limiting innate and adaptive immunity and resolving disease following respiratory syncytial virus infection. J. Virol. 84, 8790-8798 (2010).
23. Goritzka, M. et al. Alveolar macrophage-derived type I interferons orchestrate innate immunity to RSV through recruitment of antiviral monocytes. J. Exp. Med. 212, 699-714 (2015).

24. Ariotti, S. et al. Tissue-resident memory CD8 + T cells continuously patrol skin epithelia to quickly recognize local antigen. Proc. Natl. Acad. Sci. USA 109, 19739-19744 (2012).

25. Wu, T. et al. Lung-resident memory CD8 T cells (TRM) are indispensable for optimal cross-protection against pulmonary virus infection. J. Leukoc. Biol. 95, 215-224 (2014).

26. Mackay, L.K. et al. The developmental pathway for CD103(+)CD8 + tissue-resident memory Tcells of skin. Nat Immunol 14, 1294-1301 (2013).

27. Kinnear, E., Caproni, L.J. \& Tregoning, J.S. A comparison of red fluorescent proteins to model DNA vaccine expression by whole animal in vivo imaging. PLOS ONE 10, e0130375 (2015).

28. Bivas-Benita, M. et al. Airway CD8(+) T cells induced by pulmonary DNA immunization mediate protective anti-viral immunity. Mucosal Immunol. 6, 156-166 (2013).

29. Cuburu, N. et al. Intravaginal immunization with HPV vectors induces tissue-resident CD8 + T cell responses. J. Clin. Invest. 122, 4606-4620 (2012).

30. Shin, H. \& Iwasaki, A. A vaccine strategy that protects against genital herpes by establishing local memory T cells. Nature 491, 463-467 (2012).

31. Tregoning, J.S., Buffa, V., Oszmiana, A., Klein, K., Walters, A.A. \& Shattock, R.J.A. "prime-pull" vaccine strategy has a modest effect on local and systemic antibody responses to HIV gp140 in mice. PLoS ONE 8, e80559 (2013).

32. Tregoning, J.S. et al. Genetic susceptibility to the delayed sequelae of neonatal respiratory syncytial virus infection is MHC dependent. J. Immunol. 185, 5384-5391 (2010).

33. Saso, A. \& Kampmann, B. Vaccination against respiratory syncytial virus in pregnancy: a suitable tool to combat global infant morbidity and mortality? Lancet Infect. Dis. 16, e153-e163 (2016).

34. Anderson, K.G. et al. Intravascular staining for discrimination of vascular and tissue leukocytes. Nat. Protoc. 9, 209-222 (2014).

35. Donnelly, L. et al. Intravaginal immunization using the recombinant HIV-1 clade-C trimeric envelope glycoprotein CN54gp140 formulated within lyophilized solid dosage forms. Vaccine 29, 4512-4520 (2011).

36. Harker, J., Bukreyev, A., Collins, P.L., Wang, B., Openshaw, P.J. \& Tregoning, J.S. Virally delivered cytokines alter the immune response to future lung infections. J. Virol. 81, 13105-13111 (2007).

37. Cheeseman, H.M. et al. Expression profile of human Fc receptors in mucosal tissue: implications for antibody-dependent cellular effector functions targeting HIV-1 transmission. PLOS ONE 11, e0154656 (2016).

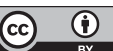

This work is licensed under a Creative Commons Attribution 4.0 International License. Theimages or other thirdparty materialin this articleareincluded in thearticle's Creative Commons license, unless indicated otherwise in the credit line; if the material is not included under the Creative Commons license, users will need to obtain permission from the license holder to reproduce the material. To view a copy of this license, visit http://creativecommons.org/licenses/by/4.0/

(C) The Author(s) 2018 